
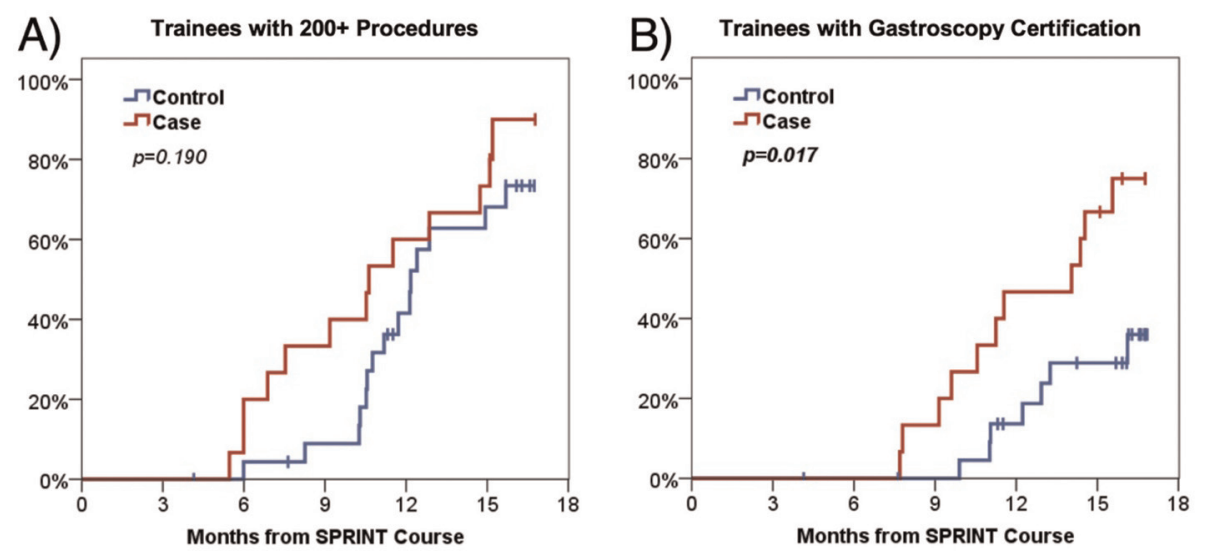

Abstract PWE-110 Figure 1

multivariable analyses performed to identify trainee-level predictors of DOPS competency.

Results Across 279 training centres, 10749 DOPS submitted for 1199 trainees were analysed. The acceptable reliability threshold $(G<0.70)$ was achieved with 3 assessors performing 2 DOPS each. Demonstrating competency in $90 \%$ of assessed items provided optimal sensitivity (90.2\%) and specificity (87.2\%) for benchmarking overall DOPS competence. This threshold was attained in the following order: 'pre-procedure' (5-24 procedures), 'endoscopic non-technical skills' and 'postprocedure' (15-99), 'management' (20-49) and 'procedure' (25-99) domain. At item-level, competency in 'proactive problem solving' (rho 0.787) and 'loop management' (rho 0.780) correlated strongest with the overall DOPS rating $(\mathrm{P}<0.001)$ and were the last to develop. The cohort achieved the DOPS benchmark score of $90 \%+$ after 20-49 procedures, which correlated with the attainment of $90 \%+$ unassisted median caecal intubation rates (Spearman rho 0.404, $\mathrm{P}<0.001$ ) (Abstract PWE109 Figure 1). On multivariable analysis, independent predictors of DOPS competence consisted of: lifetime procedure count $(\mathrm{P}<0.001)$, DOPS count $(\mathrm{P}<0.001)$, trainer specialty $(\mathrm{P}<.001)$, trainee seniority $(\mathrm{P}=0.025)$, easier case difficulty $(\mathrm{P}<0.001)$ and higher caecal intubation rate $(\mathrm{P}<.001)$.

Conclusion This study establishes milestones for competency acquisition during colonoscopy training and provides validity and reliability evidence to support colonoscopy DOPS as a competency assessment tool. Further work is required to optimise the consistency of DOPS scoring between trainer specialties to improve the reliability of DOPS assessments.

\title{
PWE-110 IMPACT OF A SIMULATION-BASED INDUCTION PROGRAMME IN GASTROSCOPY ON TRAINEE OUTCOMES
}

\footnotetext{
${ }^{1,2}$ Keith Siau*, ${ }^{2}$ James Hodson, ${ }^{3}$ Kate Edwards, ${ }^{4}$ Peter Neville, Jeff Turner, ${ }^{6}$ Susie Green, ${ }^{5}$ Amanda Beale, ${ }^{7}$ Aravinth Murugananthan, ${ }^{1,8}$ Paul Dunckley, ${ }^{1,3}$ Neil D Hawkes. ${ }^{1} J o i n t$ Advisory Group on Gastrointestinal Endoscopy (JAG), London; ${ }^{2}$ University of Birmingham, Birmingham; ${ }^{3}$ Cwm Taf UHB, Llantrisant; ${ }^{4}$ Cardiff and Vale UHB, Cardiff; ${ }^{5}$ Bristol Royal Infirmary, Bristol; ${ }^{6}$ Royal Sussex County Hospital, Brighton; ${ }^{7}$ New Cross Hospital, Wolverhampton; ${ }^{8}$ Gloucestershire Hospitals NHSFT, Gloucester
}

performance and patient outcomes. However, the durability of trainee benefit is unclear, and pre-clinical SBT remains largely unavailable in the UK. We assessed the impact of a gastroscopy induction programme (SPRINT), a two day course with review of skills handling, lesion recognition, JETS e-portfolio, report writing and a SBT curriculum, on the outcomes of novice endoscopists.

Methods In this prospective case-control study, new ST3 endoscopy trainees from three UK deaneries were enrolled to SPRINT in Sep 2017. Each received expert-led teaching with $>3$ hrs of SBT via the Surgical Science EndoSim platform. Post-course progress of attenders (cases) was compared with controls matched from the JETS training database, with follow-up until Feb 2019. Those with $>50$ procedures preceding SPRINT were excluded. Outcomes derived from JETS included: 1) unassisted D2 intubation rates, 2) time to 200 procedures, 3) time to JAG certification for independent practice. Comparisons of learning curves (D2 intubation) were made using generalised estimating equations, and of time to each milestone using Kaplan-Meier plots with Cox regression. Results After exclusions, data from 15 cases and 24 controls were analysed. Prior to the date of SPRINT, the mean lifetime procedure counts were similar in cases and controls (10 vs. 3, $\mathrm{P}=0.739$ ), with $60 \%$ of cases and $63 \%$ of controls having no experience. Learning curve analysis found no significant difference in the rate of improvement by procedure count between the groups $(p=0.205)$, with D2 intubation rates of $82 \%$ vs. $78 \%$ in cases vs. controls by the 50th post-SPRINT procedure. Over the 16-month follow up period, $87 \%$ of cases and $63 \%$ of controls reached their 200th procedure. The KaplanMeier estimated time to 200 procedures (Figure 1A) did not differ significantly between cases and controls (10.6 vs. 12.1 months; $\mathrm{P}=0.190)$. Cases performed a median of 16.2 procedures per month post-SPRINT vs. 13.8 in the control group $(\mathrm{P}=0.051)$. JAG certification was achieved in $73 \%$ of cases and $29 \%$ of controls. The median time to certification (Figure 1B) was earlier in SPRINT attenders than controls (14 vs. $>16$ months, $\mathrm{P}=0.017$ ).

Conclusions The SPRINT cohort tended to perform more procedures and achieved JAG certification earlier than controls. These data support the role for wider implementation of preclinical induction involving SBT.

\subsection{6/gutjnl-2019-BSGAbstracts.481}

Introduction Pre-clinical simulation-based training (SBT) in endoscopy is associated with improvements in trainee 